\section{PENICILLINS}

\section{Thirty Years Afier}

from a Correspondent

THE thirtieth anniversary of the introduction of penicillin into medicine was commemorated by a meeting at the Royal Society on May 20 and 21, held jointly with the Royal College of Physicians. After an interesting account by Sir Ernst Chain (Imperial College, London) of the early days in the development of penicillin at Oxford, the first day was devoted to a comprehensive survey of current scientific research relating to $\beta$-lactam antibiotics.

Recent advances in biosynthesis were reviewed by Dr N. Neuss (Eli Lilly and Co., Indianapolis) who also described his own use of ${ }^{13} \mathrm{C}$ NMR spectroscopy to determine the pathway by which acetate is incorporated into the cephalosporin $\mathrm{C}$ molecule. Professor D. H. R. Barton (Imperial College, London) discussed the mechanisms involved in the chemical conversion of penicillin sulphoxides, by way of the corresponding sulphenic acid, to cephalosporin-type molecules. Elimination of the 5-carbon fragment to give the intact $\beta$-lactam ring in excellent yield can be achieved by reaction of the dehydropyran derivative of the sulphenic acid with diazomethane, followed by zinc dust reduction.

Other topics discussed were the mode of action of the penicillins, the development of bacterial resistance and immunological problems. Penicillins kill bacterial cells by irreversibly inactivating a transpeptidase involved in the last reaction in the synthesis of cell walls. The question whether the inactivation is a consequence of the penicilloylation of transpeptidase has been investigated by $\mathrm{Dr} J$. L. Strominger (Harvard University). He reported that bacterial cells contain from two to seven components which bind radioactive penicillin, and he suggested that the rapid uptake component is likely to be responsible for the killing of bacteria; this may be the transpeptidase.

The clinical day of the meeting was concerned with the impact of antibiotics, in particular the penicillins, on the treatment of infectious disease. The dramatic decline in mortality in many instances after the introduction of penicillin was illustrated by the study on osteomyelitis reported by $\operatorname{Dr}$ P. C. Fleming (Hospital for Sick Children, Toronto). Although penicillin-resistant strains of staphylococci emerged in the 1950 s, these have been successfully treated with the newer semi-synthetic penicillins and cephalosporins.

The changing pattern in the distribution of infecting organisms and their antibiotic sensitivity was stressed by several speakers. Professor J. P.

Sandford (Texas University) discussed the increasingly important clinical problem posed by generalized Gramnegative bacillary infections, particularly bacteriaemias. These, he suggested are replacing the pneumococci and streptococci of the pre-antibiotic era as the largest single group of lifethreatening infections. Dr L. Weinstein (New England Medical Center, Boston) spoke of the altered distribution of organisms in bacterial endocarditis. There has been an increase in the number of cases caused by enterococci and a sharp rise in endocarditis caused by unusual organisms such as Salmonella and fungi.

Finally, a note of caution was sounded by Professor R. E. O. Williams (St Mary's Hospital Medical School, London), who pointed out that the body's own defence mechanisms can be damaged by antibiotic therapy. Elimination of bacterial flora from the gut, for example, could result in interspecies substitution or the risk of super-infection.

\title{
Gamma Rays under Scrutiny
}

Cosmic gamma rays are the subject of two articles in next Monday's Nature Physical Science. But the energies involved are very different in the two cases-one deals with background flux at energies of a few $\mathrm{MeV}$, the other with possible pulsed emission from pulsars at energies in excess of $10^{13} \mathrm{eV}$.

F. W. Stecker et al. are concerned principally with the gamma ray flux at energies between 1 and $6 \mathrm{MeV}$, and they take issue with previous proposals that these gamma rays are produced within the galaxy itself. Some of the data on which they have based their arguments were obtained by Vette et al. who used a scintillation counter in a small satellite to measure the spectrum of the total flux between these two energies. The crux of the matter is that Vette et al. were unable to measure the gamma ray angular distribution, and so could come to no definite conclusions about the origin of the gamma rays. Stecker et al. constructed an integral energy spectrum for the galactic disk, and show that it is unreasonable to assume that 1 to 6 $\mathrm{MeV}$ gamma rays are galactic.

They also made use of an integral measurement of the flux from the galactic disk above $100 \mathrm{MeV}$ made by Clark et al., and an upper limit of the integral flux at $50 \mathrm{MeV}$ proposed by Fichtel and Kniffen. A theoretical spectrum consistent with both the flux at $6 \mathrm{MeV}$ and the upper limit at 50 $\mathrm{MeV}$ turns out to be quite steep, but inconsistent with the Compton scattering mechanism which is thought likely to generate galactic gamma rays with
GLASS SWITCHES

\section{Ovshinsky Hot and Cold}

from our Materials Science Correspondent

FEw recent developments in technology have given rise to such vigorousoccasionally passionate - scientific argument as the electric switches based on chalcogenide glasses (Ovonic devices), originally discovered by Ovshinsky in America. There are two types of switches: the "threshold switches", which can only remain in the switched-on state while current flows, and the "memory switches" which, like computer memories, remain on or off in the passive state until kicked into the converse condition. The unique feature of the whole episode is that Ovshinsky's devices were purely empirical; he is not a trained scientist and at first did not pretend to understand how they worked. Solid-state electronics is the archetypal sciencebased field of technology, and neither physicists nor electronic engineers have

energies between about 10 and 200 $\mathrm{MeV}$. In particular, the Compton scattering spectrum is too steep to explain the data at 50 and $100 \mathrm{MeV}$, and not steep enough or of sufficient intensity to account for the spectrum between 6 and $50 \mathrm{MeV}$, which is required if the 1 to $6 \mathrm{MeV}$ gamma rays are of galactic origin. All this suggests that the 1 to $6 \mathrm{MeV}$ gamma rays are extragalactic and highly isotropic ; Stecker et al. emphasize that this will constitute a very large background on which point sources emitting gamma rays in this energy range will be superimposed. Detection of weak sources will be very difficult with the rather crude directional instruments available at present.

The same issue of Nature Physical Science also contains an attempt to detect pulses of gamma rays from pulsars by Chatterjee et al. Their technique is to look for the Cerenkov light emitted by the electron cascades generated in the atmosphere by very energetic gamma rays $\left(>10^{13} \mathrm{eV}\right)$ and to correlate the signals with the pulsed radio emission of the pulsar. The Cerenkov light is detected by large searchlight mirrors and focused onto the photocathodes of sensitive photomultipliers. Chatterjee et al. previously described the detection of significant pulsed emission from CP 0950 (Nature, 225, 839 ; 1970), but they have now repeated their observations on CP 0950 and five other pulsars and find no such effect. They conclude that their original observation must have been a chance occurrence. 